University of Nebraska - Lincoln

DigitalCommons@University of Nebraska - Lincoln

Sarcocystis cymruensis: discovery in Western Hemisphere in the

Brown rat (Rattus norvegicus) from Grenada, West Indies: redescription, molecular characterization, and transmission to IFN-Y gene knockout mice via sporocysts from experimentally infected domestic cat (Felis catus)

\author{
Fernando H. Antunes Murata \\ Beltsville Agricultural Research Center, Beltsville \\ Camila K. Cerqueira-Cézar \\ Beltsville Agricultural Research Center, Beltsville \\ Peter C. Thompson \\ Beltsville Agricultural Research Center, Beltsville \\ Keshaw Tiwari \\ St. George's University, St. George's \\ Joseph D. Mowery

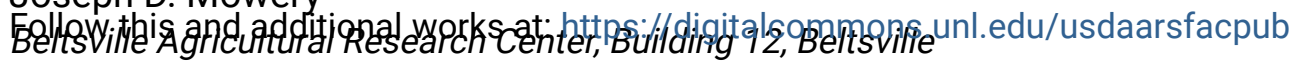

\begin{abstract}
Antunes Murata, Fernando H.; Cerqueira-Cézar, Camila K.; Thompson, Peter C.; Tiwari, Keshaw; Mowery, Soseph D.; verma, Shiv R.; Rosenthal, Benjamin M.; Sharma, Ravindra N.; and Dubey, J. P., "Sarcocystis cymruensis: discovery in Western Hemisphere in the Brown rat (Rattus norvegicus) from Grenada, West Indies: redescription, molecular characterization, and transmission to IFN- $\gamma$ gene knockout mice via sporocysts from experimentally infected domestic cat (Felis catus)" (2018). Publications from USDA-ARS / UNL Faculty. 2224.

https://digitalcommons.unl.edu/usdaarsfacpub/2224
\end{abstract}

This Article is brought to you for free and open access by the U.S. Department of Agriculture: Agricultural Research Service, Lincoln, Nebraska at DigitalCommons@University of Nebraska - Lincoln. It has been accepted for inclusion in Publications from USDA-ARS / UNL Faculty by an authorized administrator of DigitalCommons@University of Nebraska - Lincoln. 


\section{Authors}

Fernando H. Antunes Murata, Camila K. Cerqueira-Cézar, Peter C. Thompson, Keshaw Tiwari, Joseph D. Mowery, Shiv K. Verma, Benjamin M. Rosenthal, Ravindra N. Sharma, and J. P. Dubey 


\title{
Sarcocystis cymruensis: discovery in Western Hemisphere in the Brown rat (Rattus norvegicus) from Grenada, West Indies: redescription, molecular characterization, and transmission to IFN- $\gamma$ gene knockout mice via sporocysts from experimentally infected domestic cat (Felis catus)
}

\author{
Fernando H. Antunes Murata ${ }^{1}$ - Camila K. Cerqueira-Cézar ${ }^{1}$ • Peter C. Thompson ${ }^{1}$ - Keshaw Tiwari ${ }^{2}$. \\ Joseph D. Mowery ${ }^{3} \cdot$ Shiv K. Verma ${ }^{1} \cdot$ Benjamin M. Rosenthal ${ }^{1} \cdot$ Ravindra N. Sharma $^{2} \cdot$ Jitender P. Dubey $^{1}$
}

Received: 6 December 2017 / Accepted: 31 January 2018 / Published online: 19 February 2018

(C) This is a U.S. Government work and not under copyright protection in the US; foreign copyright protection may apply 2018

\begin{abstract}
Rodents are intermediate hosts for many species of Sarcocystis. Little is known of Sarcocystis cymruensis that uses the Brown rat (Rattus norvegicus) as intermediate hosts and the domestic cat (Felis catus) as experimental definitive host. Here, we identified and described Sarcocystis cymruensis in naturally infected $R$. norvegicus from Grenada, West Indies. Rats $(n=167)$ were trapped in various locations in two parishes (St. George and St. David). Microscopic, thin $(<1 \mu \mathrm{m})$ walled, slender sarcocysts were found in 11 of $156(7.0 \%)$ rats skeletal muscles by squash examination. A laboratory-raised cat fed naturally infected rat tissues excreted sporocysts that were infectious for interferon gamma gene knockout (KO) mice, but not to Swiss Webster outbred albino mice. All inoculated mice remained asymptomatic, and microscopic S. cymruensis-like sarcocysts were found in the muscles of KO mice euthanized on day 70,116, and 189 post inoculation (p.i.). Sarcocysts from infected KO mice were infective for cats at day 116 but not at 70 days p.i. By transmission electron microscopy, the sarcocyst wall was "type 1a." Detailed morphological description of the cyst wall, metrocytes, and bradyzoites is given for the first time. Additionally, molecular data on S. cymruensis are presented also for the first time. Molecular characterization of sarcocysts $18 S$ rDNA and 28S rDNA, ITS-1, and coxl loci showed the highest similarity with S. rodentifelis and S. muris. In conclusion, the present study described the natural infection of S. cymruensis in Brown rat for the first time in a Caribbean country and provided its molecular characteristics.
\end{abstract}

Keywords Sarcocystis cymruensis $\cdot$ Rattus norvegicus $\cdot$ Grenada $\cdot$ Ultrastructure $\cdot$ Phylogeny

\section{Introduction}

Sarcocystis species have worldwide distribution. Some species of Sarcocystis are pathogenic to humans and animals, and

Jitender P. Dubey

jitender.dubey@ars.usda.gov

1 United States Department of Agriculture, Agricultural Research Service, Animal Parasitic Diseases Laboratory, Beltsville Agricultural Research Center, Beltsville, MD 20705-2350, USA

2 Pathobiology Department, School of Veterinary Medicine, St. George's University, St. George's, West Indies, Grenada

3 United States Department of Agriculture, Agricultural Research Service, Electron and Confocal Microscopy Unit, Beltsville Agricultural Research Center, Building 12,

Beltsville, MD 20705-2350, USA some are zoonotic. Most Sarcocystis species are host-specific but some have a wide host range. Rodents, especially immunosuppressed, have been used to study biology of Sarcocystis. The Brown rat (Rattus norvegicus) is an intermediate host for seven or more Sarcocystis species; S. cymruensis, S. murinotechis, S. singaporensis, S. villivillosi, S. zuoi, S. zamani, and S. dispersa-like (Dubey et al. 2016). Of these, $S$. cymruensis and $S$. singaporensis are of biologic interest. With respect to $S$. cymruensis, its life cycle can be easily completed in the laboratory because domestic cat (Felis catus) is its definitive host and it has been reported that rats can act as both the intermediate and definitive host (Hu et al. 2011). For S. singaporensis, python (Python reticulatus) is the definitive host and it is highly pathogenic for the rat but host-specific. Thus, 
feed intentionally contaminated with $S$. singaporensis sporocysts has been proposed as a biological control for rats (Jäkel et al. 1996).

Sarcocystis cymruensis was named and described from the Brown rat (Rattus norvegicus) from the UK and sarcocysts were found in 36 (17.5\%) of 205 rats (Ashford 1978). Domestic cats fed naturally infected rat muscle excreted sporocysts; dogs and ferrets fed tissue did not excrete sporocysts. Rattus norvegicus became infected when fed sporocysts from experimentally infected cats, sarcocysts, sarcocysts became grossly visible 6-month post inoculation (p.i.). The parasite was not transmissible to laboratory-raised house mice (Mus musculus) (Ashford 1978). Jäkel et al. (1997) reported S. cymruensis sarcocysts in six of nine R. norvegicus in Thailand. Jäkel et al. (1996) mentioned that one Brown rat and two Roof rats (Rattus rattus frugivorous) were naturally infected with $S$. cymruensis; these rats were caught in Egypt and were used for experimental infection with $S$. singaporensis in Germany.

Hu et al. (2011) discovered S. cymruensis sarcocysts in 22 (11.6\%) of 198 wild rats (Rattus sp.) from China. Domestic cats fed naturally infected rat tissues excreted sporocysts. Sporocysts from cats were infective to $R$. norvegicus but not the outbred M. musculus. Additionally, sporocysts were detected in the lamina propria of small intestines of $R$. norvegicus fed sarcocysts from experimentally infected rats.

A new species of Sarcocystis, Sarcocystis rodentifelis, was described from Russia with a broad host range and unusual life cycle (Grikieniené et al. 1993). The Norway rat was the type intermediate host; bank vole (Clethrionomys glareolus), and the laboratory-raised house mouse (Mus musculus) acted as additional intermediate hosts. The laboratory rat acted both as the intermediate and definitive host (Grikieniené et al. 1993; Grikieniené and Kutkiené 1998). Rats fed sarcocysts excreted numerous sporocysts in feces (Grikieniené and Kutkiené 1998). Additionally, the domestic cat also acted as definitive host and $S$. rodentifelis could be transmitted transplacentally in rats (Grikieniené et al. 1993). Morphologically, $S$. rodentifelis was indistinguishable from $S$. muris and S. cymruensis (Grikieniené et al. 1993). Based on unusual characteristics, the authors proposed a subgenus Levitia for $S$. rodentifelis (Grikieniené et al. 1993).

The objective of the present paper is to document natural infection of $S$. cymruensis in Brown rats from Grenada, describe morphological and molecular characteristics, and experimental transmission.

\section{Material and methods}

\section{Sample collection}

As part of an epidemiological study of rodent pathogens, from February to July 2017, 167 rats ( 78 females, 89 males) were caught using live traps $(45 \mathrm{~cm} \mathrm{~L} \times 15 \mathrm{~cm} \mathrm{w} \times 15 \mathrm{~cm} \mathrm{~h})$ with cheese and/or various local fruits as bait. Rats were trapped in various locations in two parishes (St. George and St. David) which were densely populated compared to four remaining parishes. Attempts were made to trap the rats near residential buildings. Traps were placed in the evening and visited next morning. Traps with rats were covered with black cloth and transported to the necropsy laboratory of the St. George's University School of Veterinary Medicine and euthanized using isoflurane, and necropsied.

Samples from heart and limb muscle from 167 rats were shipped on ice by overnight delivery to the United States Department of Agriculture (USDA), Animal Parasitic Diseases Laboratory (APDL) in Beltsville, MD, USA, for protozoal testing, including Toxoplasma gondii (to be reported separately). The rats were received at APDL in 17 batches of $6,3,2,4,10,5,11,13,7,14,24,8,13,11,13,16$, and 7 samples storage in individual bags. Two to 4 days elapsed between euthanasia and receipt at APDL.

\section{Testing of rat muscle for Sarcocystis}

Samples were examined by muscle squash, pepsin digestion, histology, bioassay, transmission electron microscopy (TEM), and DNA sequencing. For muscle squashes, $1-3 \mathrm{~mm}$ of muscle from leg of 156 rats and myocardium of 167 rats were pressed between a slide and coverslip, and examined microscopically at 100-400 x magnification. For pepsin digestion, tissues (165 hearts, 154 muscles) were pooled in batches of two to ten rats (total pools 29, homogenized in saline (aqueous $0.9 \% \mathrm{NaCl}$ ), incubated in acid pepsin solution for $1 \mathrm{~h}$ at $37^{\circ} \mathrm{C}$ in a water bath, and examined for bradyzoites of cyst forming coccidian as described previously for $T$. gondii (Dubey 2010). In this process, protozoan bradyzoites are released as soon as the cyst wall is in contact with pepsin and bradyzoites can survive in the digest for several hours. The pepsin product was filtered, centrifuged, and suspended in saline, and a drop $(0.1 \mathrm{ml})$ of the digest was spread on a slide, covered with a coverslip, and examined microscopically for bradyzoites.

For TEM, six sarcocysts were teased out of muscle at APDL, fixed in $2.5 \%$ buffered glutaraldehyde and processed as described previously (Trupkiewicz et al. 2016).

\section{Bioassay of Sarcocystis in cats}

For bioassay in cats, five parasite-free (14-34 weeks old) cats from the USDA cat colony were used to obtain sporocysts; management of the cat colony has been described previously (Dubey 1995). Feces were collected daily and examined for Sarcocystis sporocysts as described (Verma et al. 2017a). Cats were euthanized between day seven to 13 p.i. The entire small intestine from each animal was removed, slit open, mucosa 
scraped, homogenized in water, and examined microscopically for sporocysts as described (Dubey et al. 2016).

\section{Bioassay of Sarcocystis in mice}

For murine bioassay, outbred albino Swiss Webster (SW) and interferon gamma gene knockout (KO) mice were used. Two SW and four KO mice were orally inoculated with sporocysts from a cat that had been fed muscle from two naturally infected rats. The number of sporocysts given to mice was unknown $(<1000)$ because the cat had excreted only a few sporocysts. Two SW and two KO mice were euthanized day 70 p.i., one KO mouse was euthanized day 116 p.i., and one KO mouse was euthanized day 189 p.i.; multiple samples of muscle were squeezed between the slide and coverslip and examined for the presence of Sarcocystis sarcocysts. Samples of muscle, heart, tongue, lung, liver, eye, spleen, kidney, brain, and intestine of all mice were fixed in $10 \%$ buffered formalin, and processed for histology. Sections were cut $5-\mu \mathrm{m}$ thick and examined microscopically after staining with hematoxylin and eosin (HE). Additionally, carcasses of mice were homogenized and digested in pepsin. The product of digestion was smeared on a slide, fixed in methanol and stained with Giemsa, and examined microscopically for bradyzoites.

Bradyzoites released from sarcocysts deriving from the $\mathrm{KO}$ mouse euthanized day 116 p.i. were fed to two KO mice. For this, a piece of muscle from the KO mouse was smashed and digested in pepsin for $5 \mathrm{~min}$ to release bradyzoites from sarcocysts. The pepsin digest was neutralized with $3 \%$ $\mathrm{NaOH}$, centrifuged, and orally inoculated into two KO mice; more than 100,000 bradyzoites were ascertained in the inocula. These two KO mice were euthanized day 6 p.i. and sections of small intestine were fixed in $10 \%$ buffered formalin. The remaining small intestine was homogenized in $50 \mathrm{ml}$ of distilled water, shaken for $1 \mathrm{~h}$ to lyse the intestinal epithelial cells, centrifuged, and suspended in distilled water, and a drop $(0.1 \mathrm{ml})$ was spread on a slide, covered with a coverslip and examined microscopically for sporocysts.

The KO mouse euthanized day 189 p.i. was heavily infected with sarcocysts. After fixing samples of internal organs, pieces of gastrocnemius muscle with hundreds of sarcocysts were chopped with scissors and saved for feeding to KO mice. After removing viscera and skin, the carcass was homogenized in blender with saline, incubated in acid pepsin at $37{ }^{\circ} \mathrm{C}$ for 15 min, neutralized with $3 \% \mathrm{NaOH}$ and Hanks Balanced Salt Solution (HBSS), centrifuged, and suspended in $3 \mathrm{ml}$ of HBSS. After counting bradyzoites, the suspension of bradyzoites (15 million per mouse) was orally inoculated into four $\mathrm{KO}$ mice. Additionally, the same four $\mathrm{KO}$ mice were fed on heavily infected mouse muscle. For this, the finely chopped muscle was left in Petri dish in the mouse cage; the mice ate all infected muscle. These four $\mathrm{KO}$ mice were euthanized on days 1-6; one on day 1 p.i., one on day 4 p.i., and two on day 6 p.i. For the mice euthanized day 1 and 4 p.i., the entire small intestine was cut in to small pieces and fixed in formalin, and paraffin-embedded sections were examined after staining with HE. From the two KO mice euthanized day 6 p.i., after removing $1-\mathrm{cm}$ piece for histology, the remaining small intestines from both mice were pooled, homogenized in $50 \mathrm{ml}$ of distilled water in a blender, shaken for $1 \mathrm{~h}$ at $37^{\circ} \mathrm{C}$, centrifuged, and suspended in $5 \mathrm{ml}$ water, and a drop of it was spread on a slide for microscopic examination. Because we did not find Sarcocystis sporocysts, the homogenate was further digested in a bleach solution (6.0\% sodium hypochlorite) for $10 \mathrm{~min}$ to dissolve most of the host tissue, centrifuged with water, and a drop $(0.1 \mathrm{ml})$ of the final $1 \mathrm{ml}$ suspension of the pellet was examined microscopically for sporocysts.

\section{Molecular characterization}

Sarcocysts excised from two naturally infected rats were individually placed in $20 \mu \mathrm{l}$ of saline $(0.85 \%)$ and frozen at $20{ }^{\circ} \mathrm{C}$ until DNA was isolated.

Genomic DNA was extracted using the DNeasy Blood and Tissue Kit (Qiagen Inc., Valencia, CA, USA) according to manufacturer's instructions. Nuclear ribosomal DNA (18S $r D N A, 28 S$ rDNA, and ITS-1) and mitochondrial cytochrome c oxidase subunit 1 (coxl) were amplified by PCR using previously published primers as described (Gjerde 2013; Gjerde 2014; Gjerde and Josefsen 2015). Each locus was amplified independently using $3-\mu l$ template, $1 \mu \mathrm{l}$ of forward and reverse primers (20 mM final concentration), $12.5 \mu \mathrm{l}$ of Taq PCR Master Mix (Qiagen, USA), and $8.5 \mu$ l of distilled water. Following an initial denaturation step of $5 \mathrm{~min}$ at $95^{\circ} \mathrm{C}, 40 \mathrm{cy}$ cles of PCR were conducted for all reactions with the following cycling conditions $\left(95^{\circ} \mathrm{C}\right.$ for $45 \mathrm{~s}, 56.5^{\circ} \mathrm{C}$ for $45 \mathrm{~s}$, and $72{ }^{\circ} \mathrm{C}$ for $1 \mathrm{~min}$ ), ending with $10 \mathrm{~min}$ at $72{ }^{\circ} \mathrm{C}$ for final extension. Excess primers and nucleotides were removed using ExoSap-It (Thermo Fisher Scientific, USA) according to manufacturer's instructions, then PCR fragments were sequenced using original amplification primers and BigDye Terminator v3.1 cycle sequencing chemistry (Applied Biosystems, Foster City, CA, USA). Sequencing products were analyzed on an ABI 3730 Sequencer.

Sequencing chromatograms were examined in Geneious R10.2.3 (Biomatters Limited, New Zealand), and consensus sequences for each sample were generated only from areas where both forward and reverse sequencing were present. Consensus sequences were then compared to other Sarcocystis sequences deposited in GenBank (https://www. ncbi.nlm.nih.gov/genbank).

In order to establish phylogenetic relationships between Sarcocystis species, sequences were aligned using the Geneious v.10.2.3 MUSCLE plug-in and neighbor-joining trees were built with the HKY substitution model in the Geneious v.10.2.3 Tree Builder. Consensus trees were constructed from 

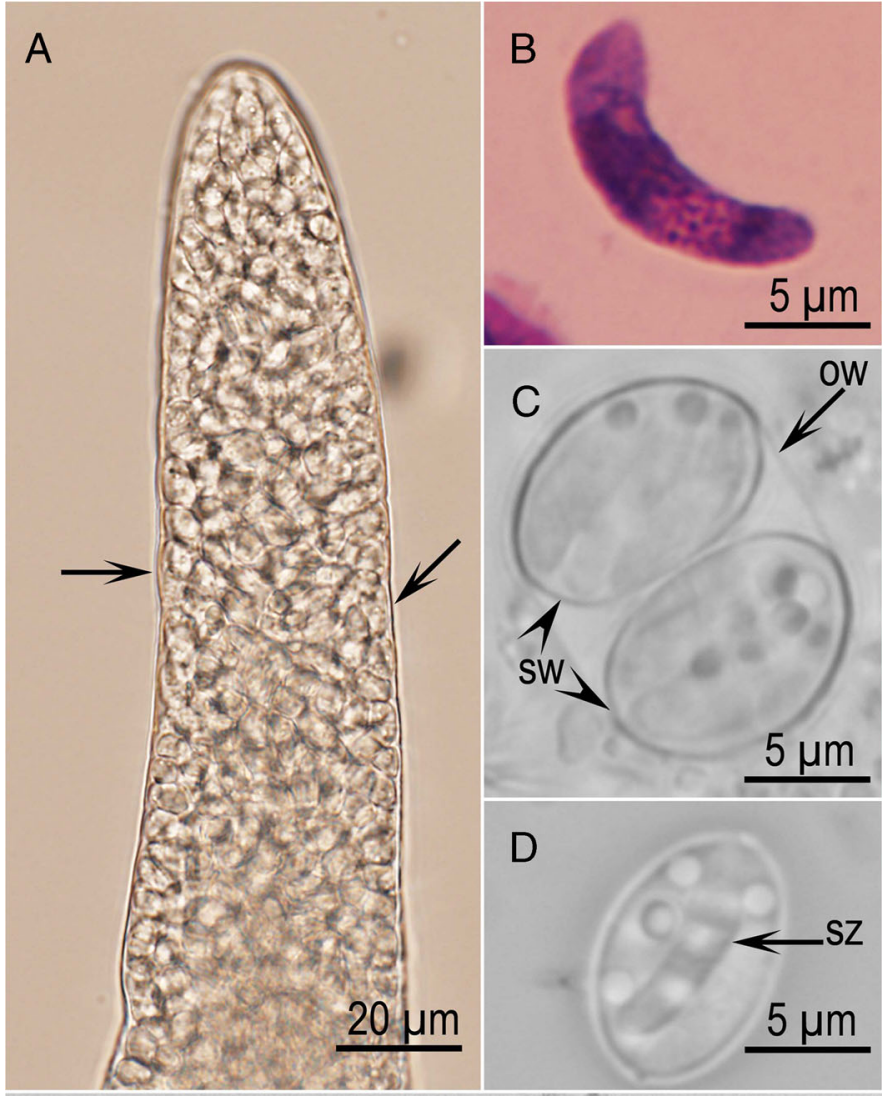

E
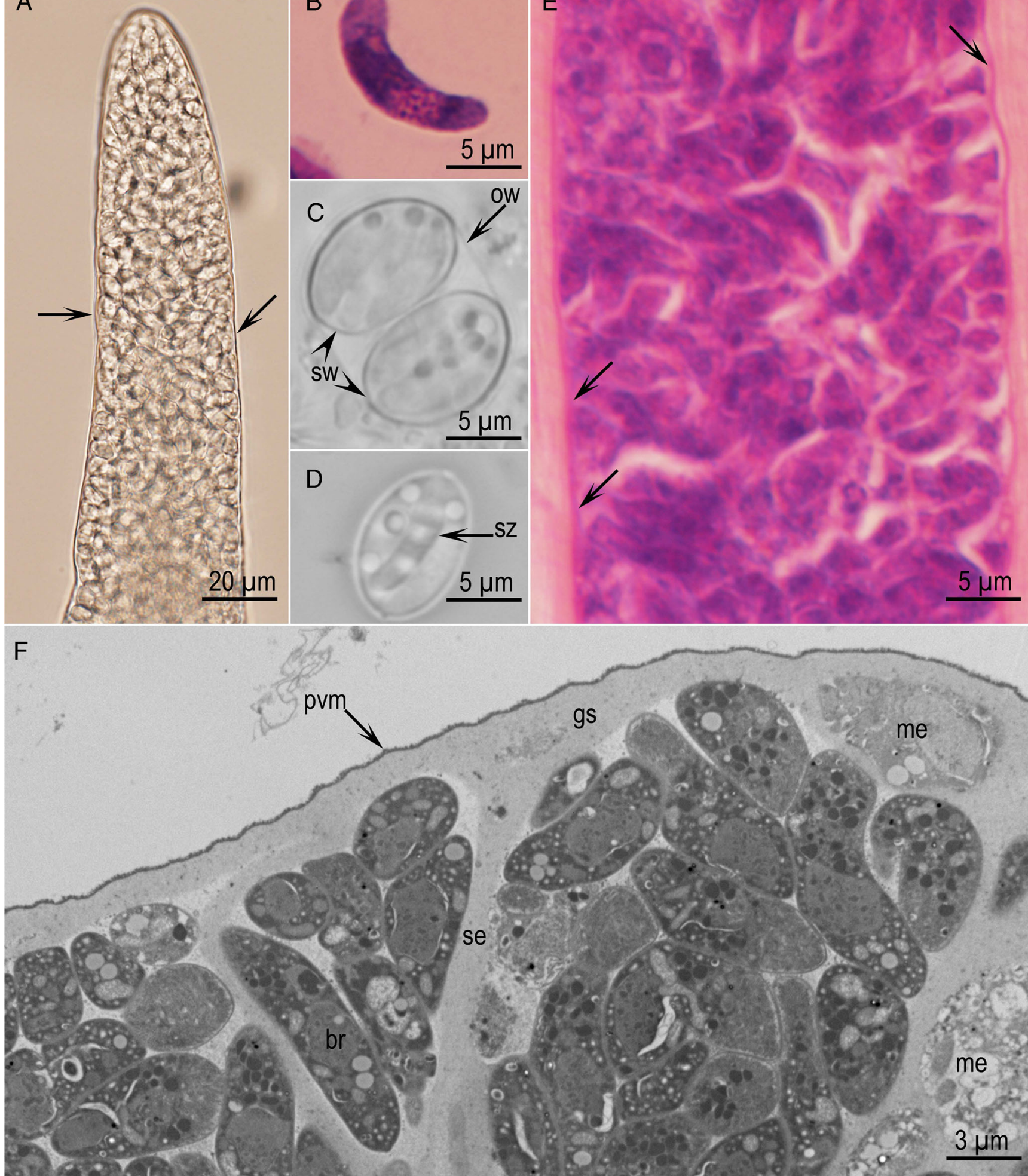

Fig. 1 Sarcocystis cymruensis stages. a Part of sarcocyst from leg muscle of a naturally infected rat \#7. The sarcocyst wall is thin (arrows) and encloses bradyzoites. Unstained. b Bradyzoite in pepsin digest of muscle. Giemsa stain. c Sporulated oocyst with a thin oocyst wall (ow), enclosing two sporocysts with prominent walls (sw). Unstained. d Sporocyst containing sporozoites (sz). Unstained. e. Longitudinal section of a sarcocyst in skeletal muscle of $\mathrm{KO}$ mouse, 70 days p.i. Note thin sarcocyst wall (arrows). Hematoxylin and eosin stain. f TEM of a part of sarcocyst wall. Note thin parasitophorous vacuolar membrane (pvm), smooth ground substance layer (gs), thick septa (se), metrocytes (me), and bradyzoites (br). Cyst \#1 
alignments of 1118 sites from 23 taxa at the $18 S r D N A$ locus and 760 sites from 14 taxa at the $28 S \mathrm{rDNA}$ locus. Alignments were constrained by the length of the $S$. cymruensis sequence at the $18 S$ $r D N A$ locus and the $S$. rodentifelis sequence at the $28 S$ rDNA locus. Insertions or deletions (indels) accounted for the difference between the length of alignment and the length of the shortest sequence. These indels were ignored for the purposes of tree building. Support for the resultant trees was assessed using 1000 bootstrap replicates.

\section{Ethics}

Research at the Beltsville laboratory was performed in accordance with Animal Protocols \#15-018 Toxoplasmosis in cats, approval date May 19, 2015, and \#15-017 Toxoplasmosis in mice, approval date May 19, 2015, Chair for both protocols Dr. Ransom Baldwin, Beltsville Institutional Animal Care and Use Committee (BAACUC). These protocols are good for 3 years and can be amended.

The project (Detection of zoonotic pathogens in brown rats (Rattus norvegicus) in Grenada) was approved by the Institutional Animal Care and Use Committee (IACUC \#16009-R, approval date May 23, 2016, Chair Dr. Karin Guerrero) of the St. George's University, Grenada.

\section{Results}

Sarcocysts were found in fresh muscle squashes of skeletal muscle of $11(7.0 \%)$ of 156 rats but not in any heart; infected rats were captured in St. Georges (6 rats) and St. David (5 rats). In fresh squashes, sarcocysts were slender, up to

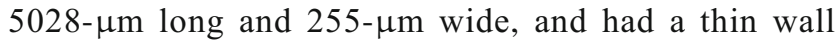
(Fig. 1a). Bradyzoites (Fig. 1b) were detected microscopically in pepsin digests in six pools of muscles derived from 123 rats. Histologically, sarcocysts were thin walled in sections of muscle stained with HE (Fig. 1e).

The cat fed naturally infected rat tissues excreted few sporulated oocysts and sporocysts 6 days p.i. (Fig. 1c, d). The mice inoculated with sporocysts from cat remained asymptomatic. Sarcocysts were detected microscopically in muscle squashes of two KO mice but not in two SW mice euthanized day 70 p.i. Sarcocystis bradyzoites were detected in pepsin digests and HE-stained sections of muscles from the two KO mice. Neither bradyzoites in carcass digests nor sarcocysts in HEstained sections were found in muscles of SW mice. Bradyzoites in stained smears averaged $16.3 \times 3.3 \mu \mathrm{m}$ with a range of $12.5-19.5 \times 1.9-4.8 \mu \mathrm{m}(n=35)$. No Sarcocystis sporocysts were found in feces or small intestine of the two cats fed muscle of two KO and two SW mice euthanized day 70 p.i.

Numerous mature slender sarcocysts were detected in the $\mathrm{KO}$ mouse euthanized day 116 p.i. Live bradyzoites were $12.1 \times$ $3.4 \mu \mathrm{m}(10.5-13.5 \times 2.7-4.5, n=30)$. Sarcocystis sporocysts were found in the small intestine of the cat fed mouse carcass euthanized on day 116 p.i. No Sarcocystis sporocysts were found in the small intestine of six $\mathrm{KO}$ mice fed bradyzoites.

Numerous sarcocysts were found in muscles of the KO euthanized day 189 p.i. This KO mouse remained asymptomatic. Sarcocysts were mature, confined to skeletal muscle, and were not grossly visible. The muscle mass seems to be increased although there was no inflammation (Fig. 2). Numerous sarcocysts were present in histologic sections and millions of mature bradyzoites were present in the carcass digest.

Six sarcocysts were studied ultrastructurally. TEM examination confirmed that the sarcocyst wall was thin (Figs. 1f, 3ad). The parasitophorous vacuolar membrane (pvm) was lined by a $40-60-\mathrm{nm}$ thick layer with variable density (edl). The pvm had 100-nm blebs (evaginations) with a stalk. Depending on the plane of section, some blebs appeared hyphenated (Fig. 3c, d). The pvm was invaginated at irregular distances (Fig. 3c) that appeared like pits on the wall; the edl had thinned out in pits. Beneath the pvm was the smooth ground substance layer (gs). The gs was usually 400-500nm thick, lacked microtubules, but had few granules (gr). The character of the cyst wall at 116 and 189 days p.i. was identical. Sarcocysts studied were mature and contained numerous bradyzoites and few metrocytes, juxtaposed with gs. The metrocytes (me) were irregular in shape, divided by endodyogeny, and had few organelles (Fig. 4). Longitudinally cut bradyzoites were $9.9 \times 3.0(7.7-11.2-$ 2.7-3.5) $\mu \mathrm{m}$, and contained a conoid, micronemes (mn),

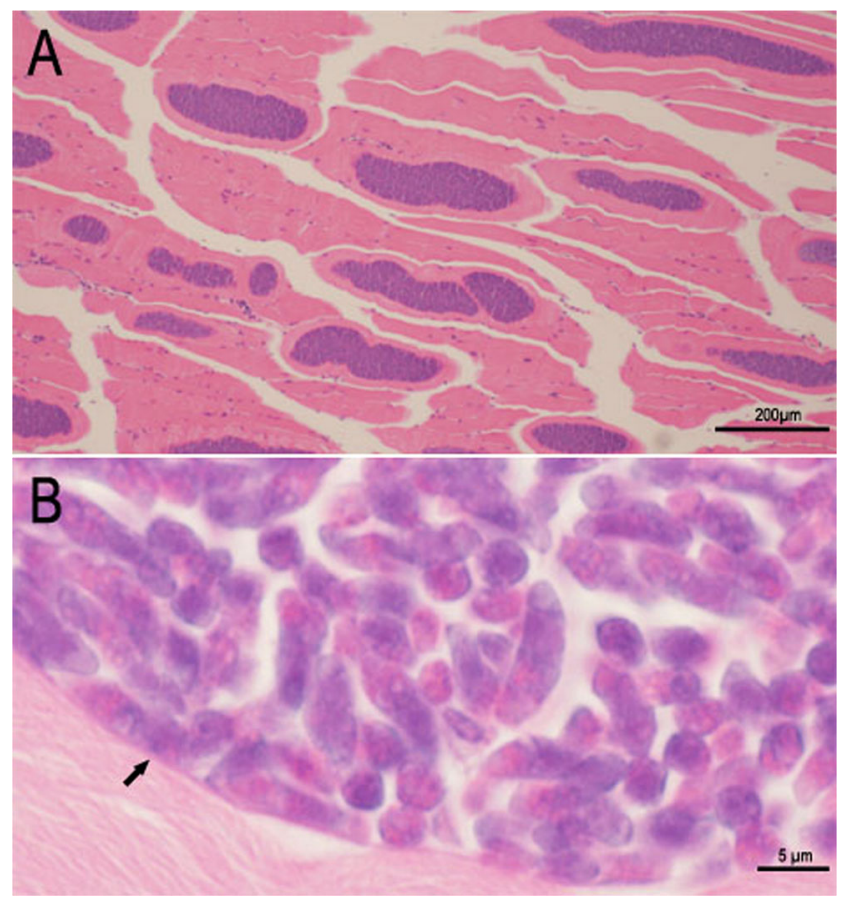

Fig. 2 Sarcocystis cymruensis sarcocysts in gastrocnemius muscle of a KO mouse 189 days post inoculation. Hematoxylin and eosin stain. (a) Note numerous mature sarcocysts. b Higher magnification of a sarcocyst. Note thin cyst wall (arrow) enclosing mature bradyzoites 


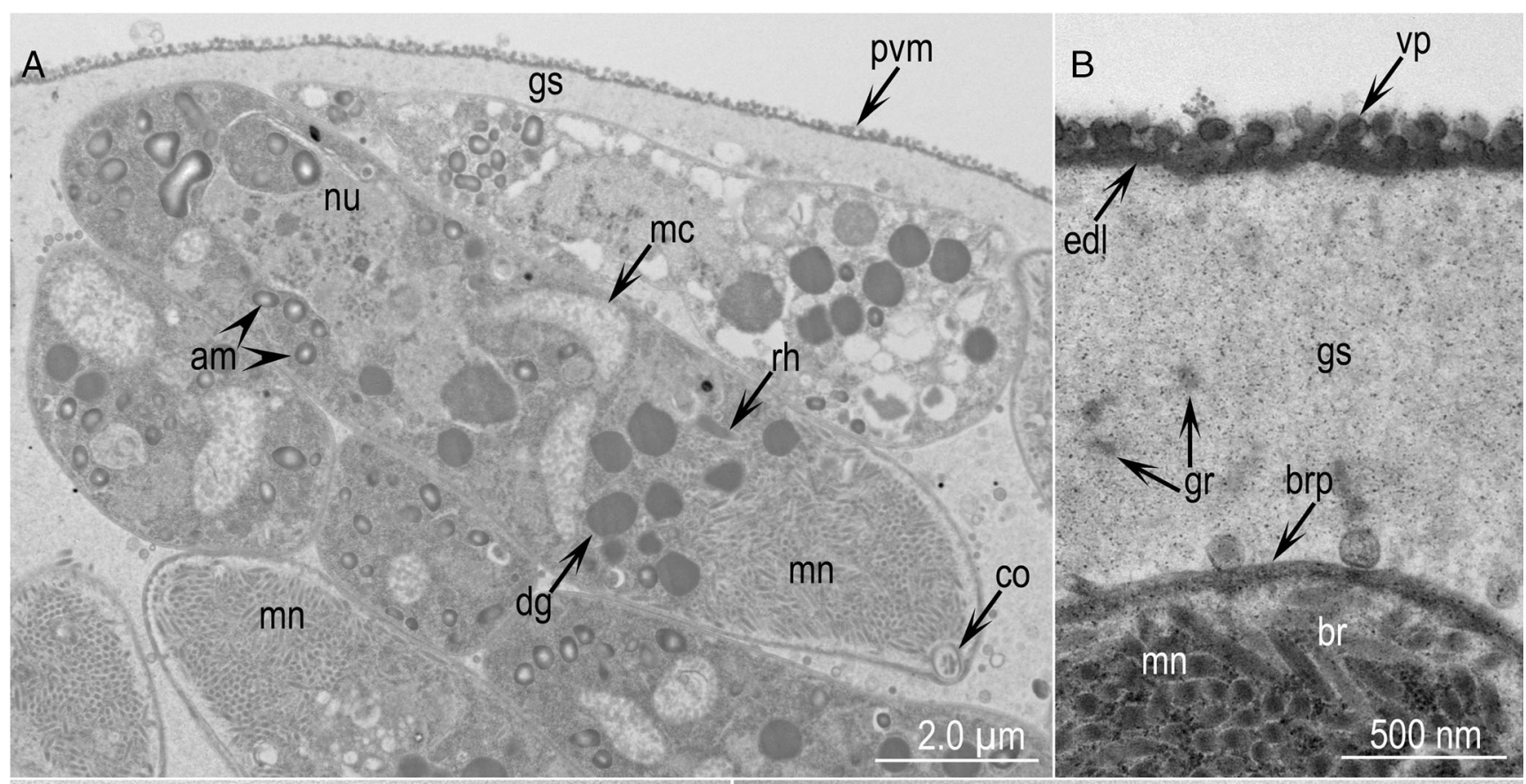

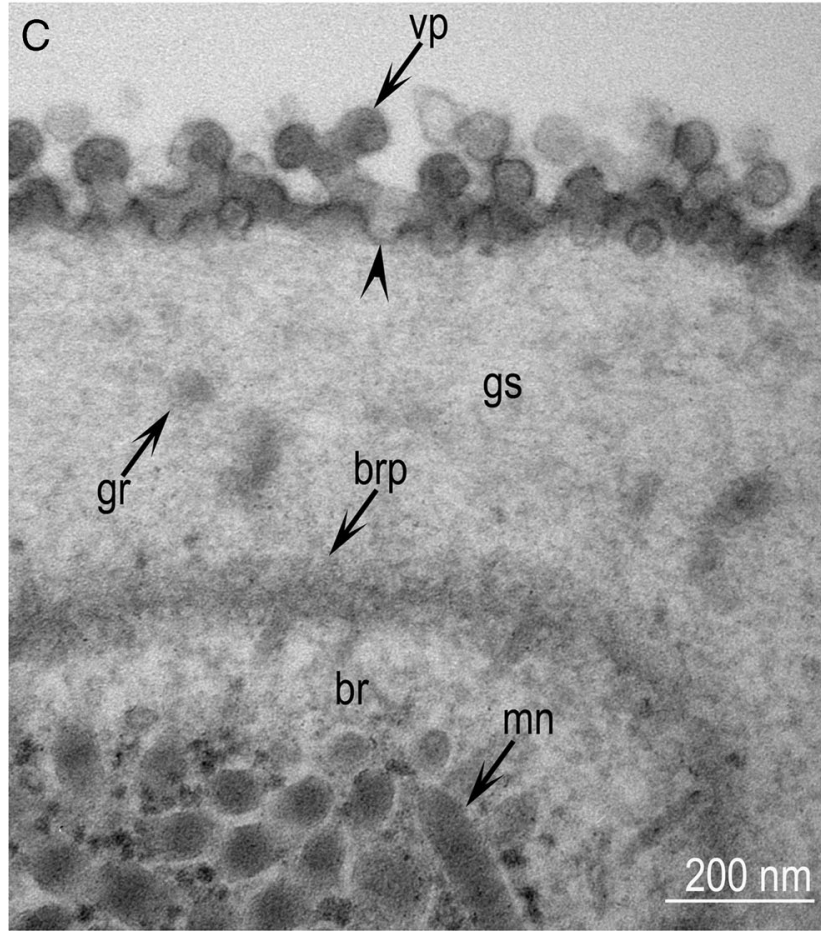

Fig. 3 TEM of S. cymruensis sarcocysts. Note cyst wall (cw), parasitophorous vacuolar membrane (pvm), electron dense layer (edl), villar protrusion (vp) ground substance layer (gs), bradyzoite pellicle (brp), bradyzoite (br), nucleus (nu), microneme (mn), amylopectin (am),

rhoptries (rh), dense granules (dg), amylopectin granules (am), nucleus (nu), and a mitochondrion (mc) (Fig. 5). The pellicle of bradyzoite was thickened at the conoidal end (Fig. $5 b$ ). The micronemes were about $320 \times 60 \mathrm{~nm}$ and distributed both haphazardly and in rows (Fig. 3d, 5b). Only one rhoptry was seen in any bradyzoite. There were several dense granules (dg), often concentrated at the conoidal half of the bradyzoite.

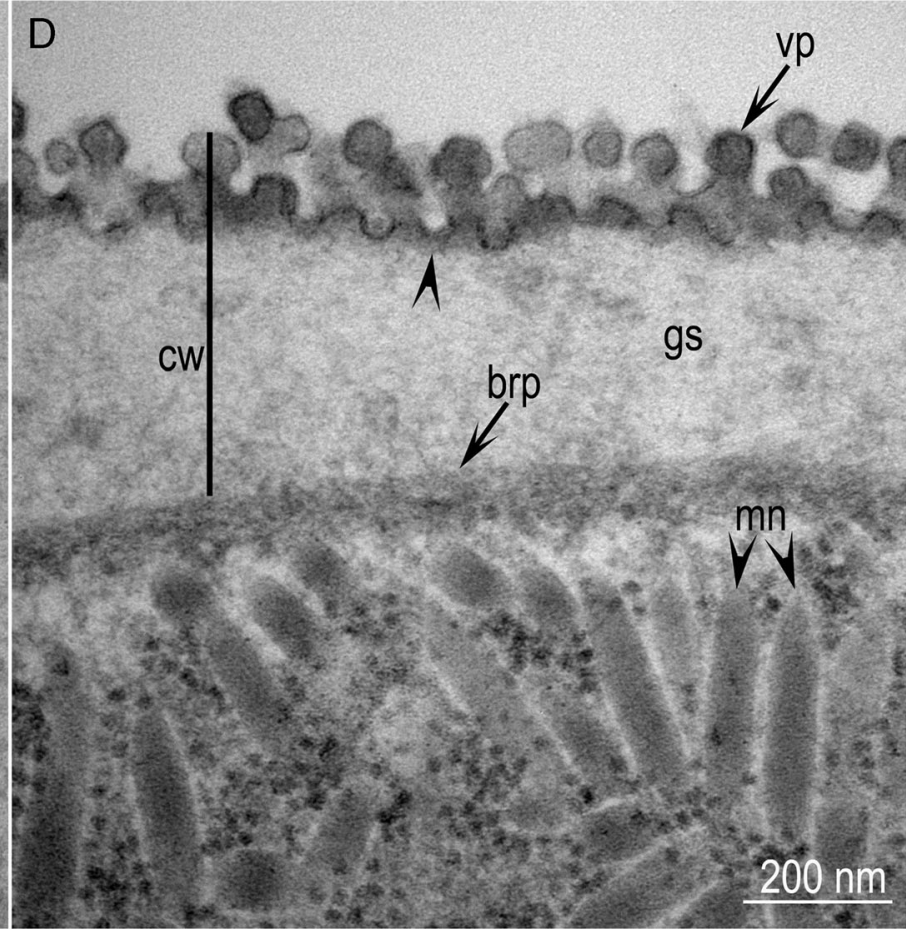

dense granules (dg), rhoptry (rh), conoid (co), and mitochondrion (mc). a A longitudinally cut and parts of several other bradyzoites. Cyst \#2. b Note bleb-like vp, gs with few granules (gr). c, d Note pits (arrowheads) the pvm. Cyst \#2

Several amylopectin granules (am) were concentrated in the distal half the bradyzoite. The nucleus was subterminal.

\section{Molecular analyses}

Partial DNA sequences were successfully acquired from $18 S$ $r D N A, 28 S$ rDNA, ITS-1, and cox1. At all loci, DNA 


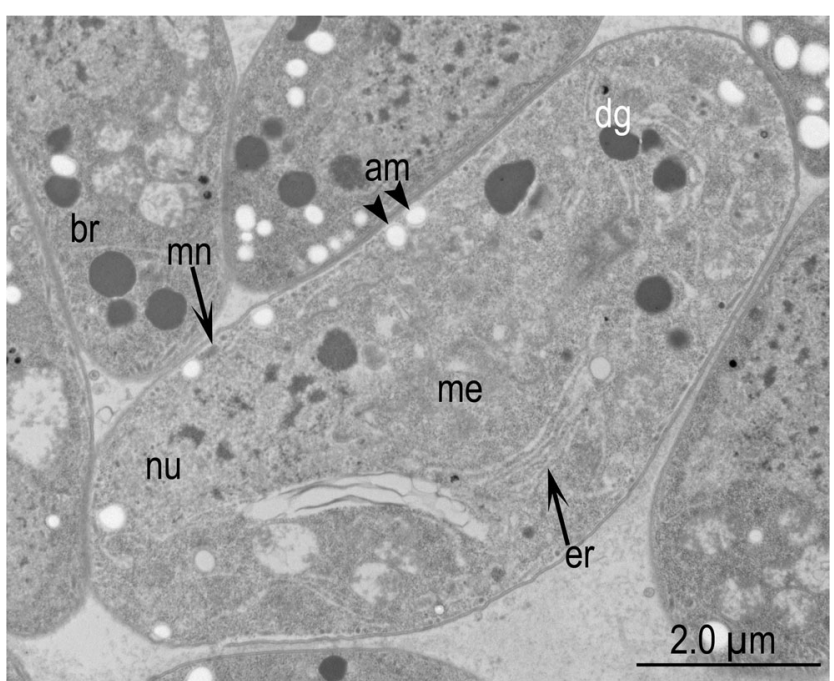

Fig. 4 TEM of $S$. cymruensis showing a metrocyte (me) and parts of 4 bradyzoites (br). Note the metrocyte (me) has no rhoptry, only 1 microneme $(\mathrm{mn}), 6$ dense granules $(\mathrm{dg})$, smooth endoplasmic reticulum (er), few amylopectin granules (am), and a large nucleus (nu). Cyst \#3

sequences were identical from sarcocysts isolated from two different mice. These sequences were submitted to NCBI GenBank with accession numbers MG564723 (18S rDNA), MG564724 (28SrDNA), MG582596 (ITS-1), and MG571085 (coxl) and designated as $S$. cymruensis from naturally infected $R$. norvegicus from Grenada. BLAST searches revealed that sequences from the $18 S$ rDNA (987 bp in length) and $28 S$ rDNA (1511 bp in length) ribosomal DNA were most similar to published sequences from S. muris (KC877996 and AF012883, respectively) and $S$. rodentifelis (AY015111 and AF513496). Sarcocystis cymruensis $18 \mathrm{SrDNA}$ and $28 \mathrm{SrDNA}$ sequences were 100 and $96.8 \%$ identical to $S$. muris sequences, respectively, while $S$. rodentifelis sequences shared $99.5 \%$ and $99.3 \%$ identity with them; $S$. rodentifelis sequences were considerably shorter at the $28 S \mathrm{rDNA}$ locus. BLAST searches determined that coxl (998 bp) shared $95.5 \%$ identity with S. speeri (KT207461) or S. lutrae (KM657808); there were no published coxl sequences for $S$. muris or S. rodentifelis in GenBank. While ITS-1 flanking sequences corresponding to the last 71 bases of $18 \mathrm{~S}$ rDNA and first 62 bases of 5.8S rDNA were similar to those from S. lutrae ( $93 \%$ identity), the ITS-1 sequences (743 bp) generated from S. cymruensis did not return any significant BLAST hits, despite searching various subsections of the molecule.

Phylogenetic trees for both $18 S \mathrm{rDNA}$ and $28 S \mathrm{rDNA}$ showed 100\% bootstrap support for S. cymruensis, S. muris, and $S$. rodentifelis belonging to an independent clade in the Sarcocystis tree (Fig. 6a, b). There are no coxl or ITS-1 sequences for S. muris or S. rodentifelis in GenBank, negating complete phylogenetic analysis across all loci sequenced.

\section{Discussion}

This is the first report of $S$. cymruensis in the Americas. The $7.0 \%$ prevalence in the present study is lower than $11.6 \%$
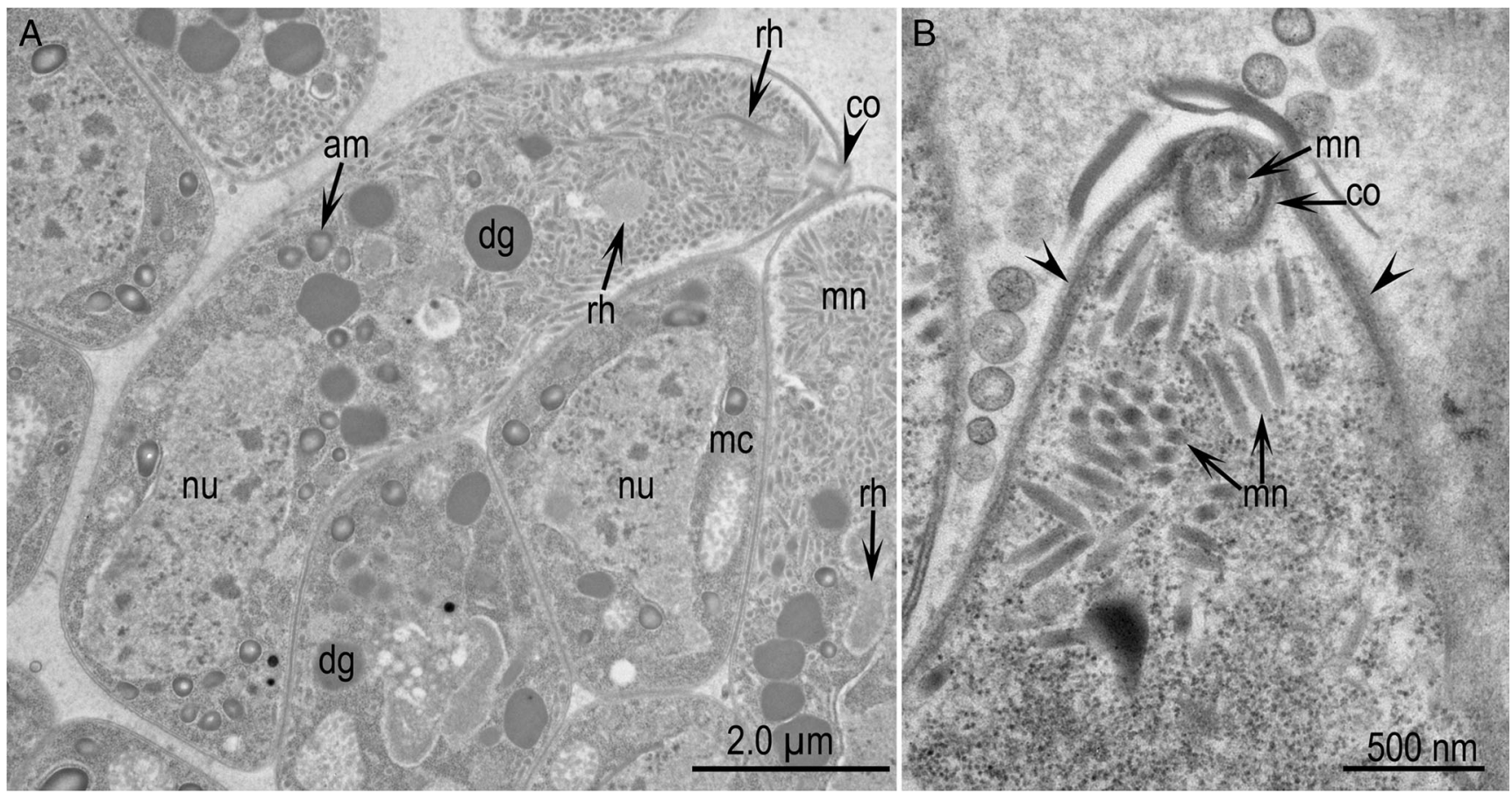

Fig. 5 TEM of S. cymruensis bradyzoites. a A longitudinally cut and parts of other bradyzoites. Note nucleus $(\mathrm{nu})$, microneme $(\mathrm{mn})$, amylopectin (am), dense granules (dg), rhoptry (rh), conoid (co), and mitochondrion (mc). b Conoidal end of a bradyzoite showing details of conoid and micronemes. Note thickening of the bradyzoite pellicle (arrowheads). Cyst \#3 


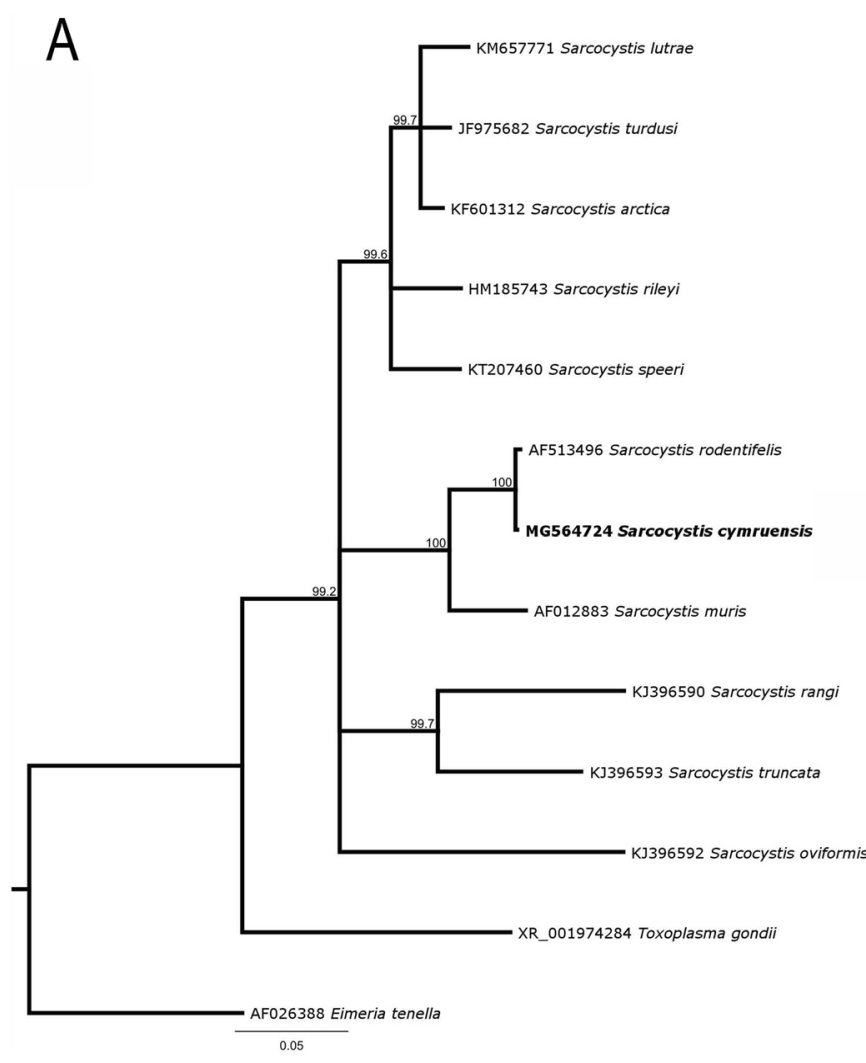

Fig. 6 Phylogenetic relationships among Sarcocystis species based on a $28 S$ rDNA ribosomal DNA sequences and b $18 S$ rDNA ribosomal DNA sequences. Neighbor-joining trees for each locus were constructed from

prevalence in China (Hu et al. 2011) and $17.5 \%$ prevalence in the UK (Ashford 1978). The distribution of definitive host may be important concerning prevalence. The natural definitive host for $S$. cymruensis remains unknown; cat is the experimental definitive host.

The present study confirmed the separate identity of S. cymruensis although its sarcocyst is morphologically similar to $S$. muris of mice. Sarcocystis cymruensis was not transmissible to immunocompetent house mouse (Ashford 1978); here, we have confirmed this. Additionally, we demonstrated that S. cymruensis is transmissible to immunodeficient $\mathrm{KO}$ mice, as in S. muris (Al-Kappany et al. 2013). The KO mice have proved very useful in studying the biology of Sarcocystis neurona, a parasite that causes fatal neurological disease in horses and many other mammals (Dubey et al. 2016). Recently, some species of Sarcocystis from snakes and red-tailed hawks (Verma et al. 2016; Verma et al. 2017b, c) induced schizont-associated neurological disease in KO mice. In the present study, the KO mice fed S. cymruensis sporocysts remained asymptomatic but developed sarcocysts. The sarcocysts in KO mice euthanized 70 days p.i. contained bradyzoites but were apparently not infectious to cat. To maximize chances of detection of few sporocysts, the intestinal mucosa was treated with undiluted bleach $(5.25 \%$ sodium hypochlorite) to dissolve host tissue (Dubey et al. 2016). The period prior to becoming infectious for $S$. cymruensis is

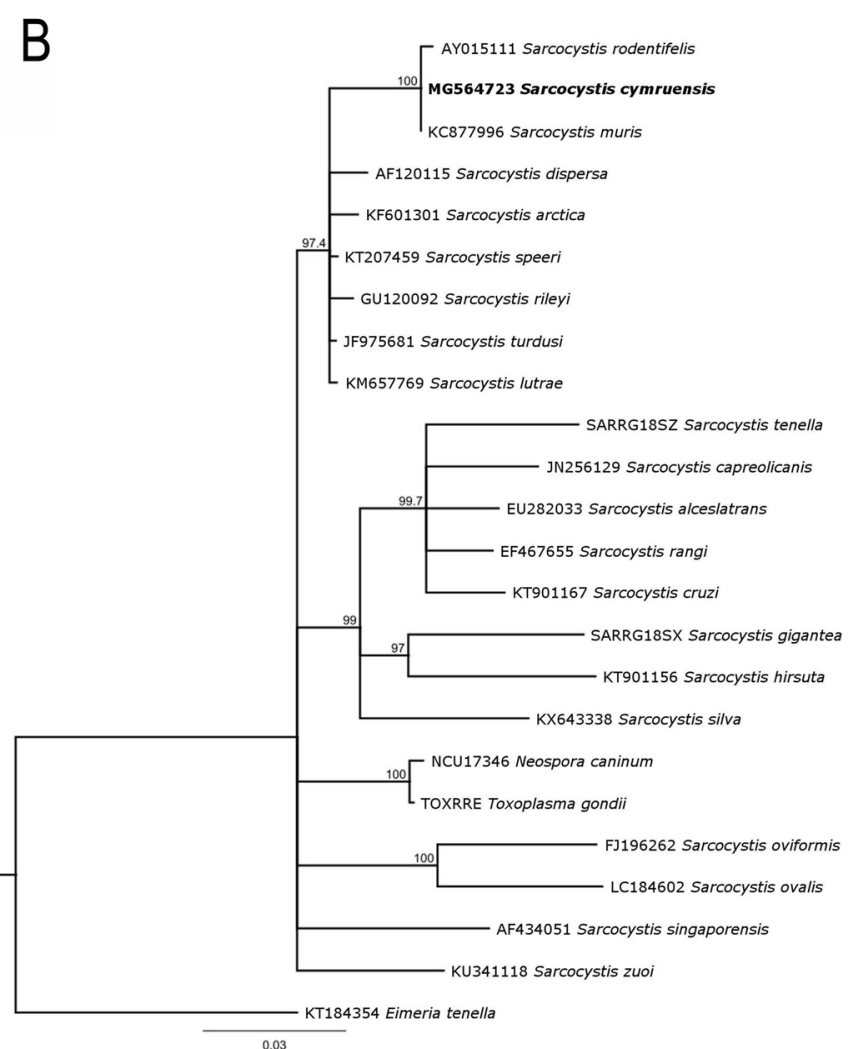

HKY genetic distances with 1000 bootstrap replicates. In both trees, an independent clade is formed with $100 \%$ bootstrap support which contains S. cymruensis, S. muris, and S. rodentifelis

unknown; in previous studies (Ashford 1978; Hu et al. 2011), cats were fed tissues with grossly visible sarcocysts from naturally infected rats whose infection duration was unknown. We chose to euthanize $\mathrm{KO}$ mice at 70,116 , and 189 days p.i. because in most sarcocyst species, sarcocysts become mature at 70 days p.i. (Dubey et al. 2016), but some species take longer. Sarcocystis muris sarcocysts became infectious for cats beginning at 76 days p.i., while sarcocysts at 65 days p.i. were not infectious (Ruiz and Frenkel 1976). Thus, the morphologic appearance of bradyzoites may not equate with infectivity. In the present study of KO mice euthanized day 70 p.i., intact, motile bradyzoites were seen in pepsin digest, confirmed by TEM, but were not infectious for cats. Infectivity in cats was only seen after day 116 p.i. in KO mice. Therefore, the range of the development of infectivity is between 70 and 116 days.

In the present study, $\mathrm{KO}$ mice acted as intermediate hosts but not as definitive hosts for S. cymruensis from Grenada. These findings are different than those obtained with $S$. rodentifelis in rats (Grikieniené et al. 1993) and S. cymruensis in rats (Hu et al. 2011). In the present study, four KO mice had ingested millions of free bradyzoites and hundreds of intact sarcocysts from a very heavily infected KO mouse euthanized 189 days p.i. The KO mice were euthanized on days 1,4 , and 6 ; the prepatent period for $S$. cymruensis is $<8$ days. We could not find gamonts, oocysts, or sporocysts in $\mathrm{KO}$ mice euthanized $1-6$ days p.i. In 
addition to examination of histological sections, the entire small intestines were digested in bleach to dissolve the host tissue. Whether these findings are related to different intermediate hosts (rats versus $\mathrm{KO}$ mice) or to different parasites is uncertain and requires further studies.

The results of the present study supplement the preliminary description of sarcocyst and bradyzoites by Ashford (1978) and $\mathrm{Hu}$ et al. (2011). The ultrastructure of cyst wall is a useful criterion in taxonomy of Sarcocystis for species within a given host (Dubey et al. 2016). Based on structure, the sarcocyst wall was grouped into 42 types with subdivisions within a type. The simplest of all wall types, "type 1, " occurs in S. muris and several other species; it was further subdivided in 'type 1a-h.' In type 1a, the wall has bleb-like protrusions that have a stalk as described in detail for S. muris (Sheffield et al. 1977). Dubey et al. (2016), in their review considered S. cymruensis as "type 1c" because in the paper by Ashford (1978), the blebs were hyphenated. Results of the present study showed that the hyphae appearance is because of angle of sectioning. Therefore, S. cymruensis wall should be regarded as belonging to type 1a, the same as in $S$. muris. We have also supplemented the morphologic description of S. cymruensis bradyzoites.

We also provide the first molecular data from $S$. cymruensis, revealing it to be a close relative to $S$. muris and $S$. rodentifelis. While $S$. cymruensis $18 S \mathrm{rDNA}$ sequences were identical to that of $S$. muris, $28 S \mathrm{rDNA}$ sequences were more similar to $S$. rodentifelis sequence. The coxl sequence most closely resembled sequences from $S$. lutrae and S. speeri, but was limited in its phylogenetic utility with absence of published coxl sequences for $S$. muris or S. rodentifelis. At the $18 S$ rDNA and $28 S$ rDNA loci, S. cymruensis had $97.3 \%$ and $93.0 \%$ identity with S. lutrae, respectively, placing $S$. cymruensis, S.muris, and $S$. rodentifelis in a separate clade of the Sarcocystis tree. Additional sequencing would be required to determine which two species in the S. cymruensis, S. muris, and $S$. rodentifelis clade are most closely related and divergence times among them. The combined divergence at the $18 \mathrm{~S}$ and $28 \mathrm{~S}$ loci and the lack of infectivity in immunocompetent house mice supports $S$. cymruensis as probably being distinct from $S$. muris.

Based on the molecular data from the present paper and review of literature, there is uncertainty concerning taxonomic status of $S$. cymruensis/S. rodentifelis and additional studies are required to resolve this issue.

Acknowledgements This research was supported in part by an appointment to the Agricultural Research Service (ARS) Research Participation Program administered by the Oak Ridge Institute for Science and Education (ORISE) through an interagency agreement between the U.S. Department of Energy (DOE) and the U.S. Department of Agriculture (USDA). ORISE is managed by ORAU under DOE contract number DESC0014664.

\section{Compliance with ethical standards}

Disclaimer All opinions expressed in this paper are the author's and do not necessarily reflect the policies and views of USDA, ARS, DOE, or ORAU/ORISE.

\section{References}

Al-Kappany YM, Abu-Elwafa SA, Hilali M, Rosenthal BM, Dunams DB, Dubey JP (2013) Experimental transmission of Sarcocystis muris (Apicomplexa: Sarcocystidae) sporocysts from a naturally infected cat (Felis catus) to immunocompetent and immunocompromised mice. J Parasitol 99(6):997-1001. https://doi.org/10.1645/13-274.1

Ashford RW (1978) Sarcocystis cymruensis n. sp., a parasite of rats Rattus norvegicus and cats Felis catus. Ann Trop Med Parasitol 72(1):37-43. https://doi.org/10.1080/00034983.1978.11719277

Dubey JP (1995) Duration of immunity to shedding of Toxoplasma gondii oocysts by cats. J Parasitol 81(3):410-415. https://doi.org/ $10.2307 / 3283823$

Dubey JP (2010) Toxoplasmosis of animals and humans, 2nd edn. CRC Press, Boca Raton, Florida, pp 1-313

Dubey JP, Calero-Bernal R, Rosenthal BM, Speer CA, Fayer R (2016) Sarcocystosis of animals and humans, 2nd edn. CRC Press, Boca Raton, Florida, pp 1-481

Gjerde B (2013) Phylogenetic relationships among Sarcocystis species in cervids, cattle and sheep inferred from the mitochondrial cytochrome c oxidase subunit I gene. Int J Parasitol 43(7):579-591. https://doi.org/10.1016/j.ijpara.2013.02.004

Gjerde B (2014) Morphological and molecular characteristics of four Sarcocystis spp. in Canadian moose (Alces alces), including Sarcocystis taeniata $\mathrm{n}$. sp. Parasitol Res 113(4):1591-1604. https:// doi.org/10.1007/s00436-014-3806-Z

Gjerde B, Josefsen TD (2015) Molecular characterisation of Sarcocystis lutrae n. sp. and Toxoplasma gondii from the musculature of two Eurasian otters (Lutra lutra) in Norway. Parasitol Res 114(3):873886. https://doi.org/10.1007/s00436-014-4251-8

Grikieniené J, Kutkiené L (1998) New experimental data on the laboratory rat as a definitive host of Sarcocystis rodentifelis. Acta Zool Lituanica Parasitologia 8(1):121-124. https://doi.org/10.1080/ 13921657.1998.10541451

Grikieniené J, Arnastauskiené T, Kutkiené L (1993) On some disregarded ways of sarcosporidians circulation and remarks about systematics of the genus Sarcocystis Lankester, 1882 with the description of the new species from rodents. Ekologija 1:16-24 (In Russian)

Hu JJ, Liao JY, Meng Y, Guo YM, Chen XW, Zuo YX (2011) Identification of Sarcocystis cymruensis in wild Rattus flavipectus and Rattus norvegicus from People's Republic of China and its transmission to rats and cats. J Parasitol 97(3):421-424. https://doi. org/10.1645/GE-2633.1

Jäkel T, Burgstaller H, Frank W (1996) Sarcocystis singaporensis: studies on host specificity, pathogenicity, and potential use as a biocontrol agent of wild rats. J Parasitol 82(2):280-287. https://doi.org/10. $2307 / 3284161$

Jäkel T, Khoprasert Y, Sorger I, Kliemt D, Seehabutr V, Suasa-ard K, Hongnark S (1997) Sarcosporidiasis in rodents from Thailand. J Wildl Dis 33(4):860-867. https://doi.org/10.7589/0090-3558-33.4.860

Ruiz A, Frenkel JK (1976) Recognition of cyclic transmission of Sarcocystis muris by cats. J Infect Dis 133:409-418

Sheffield HG, Frenkel JK, Ruiz A (1977) Ultrastructure of the cyst of Sarcocystis muris. J Parasitol 63(4):629-641. https://doi.org/10. 2307/3279561

Trupkiewicz JG, Calero-Bernal R, Verma SK, Mowery J, Davison S, Habecker P, Georoff TA, Ialeggio DM, Dubey JP (2016) Acute, fatal 
Sarcocystis calchasi-associated hepatitis in Roller pigeons (Columbia livia f. dom.) at Philadelphia Zoo. Vet Parasitol 216: 52-58. https://doi.org/10.1016/j.vetpar.2015.11.008

Verma SK, Lindsay DS, Rosenthal BM, Dubey JP (2016) Ancient, globally distributed lineage of Sarcocystis from sporocysts of the eastern rat snake (Pantherophis alleghaniensis) and its relation to neurological sequalae in intermediate hosts. Parasitol Res 115(7):2697-2704. https://doi.org/10.1007/s00436-016-5086-2

Verma SK, Lindsay DS, Grigg ME, Dubey JP (2017a) Isolation, culture and cryopreservation of Sarcocystis species. Curr Protoc Microbiol 45:20D
Verma SK, Lindsay DS, Mowery JD, Rosenthal BM, Dubey JP (2017b) Sarcocystis pantherophisi n. sp., from eastern rat snakes (Pantherophis alleghaniensis) as definitive hosts and interferon gamma gene knockout mice as experimental intermediate hosts. J Parasitol 103(5):547-554. https://doi.org/10. $1645 / 17-2$

Verma SK, Von Dohlen AR, Mowery JD, Scott D, Rosenthal BM, Dubey JP, Lindsay DS (2017c) Sarcocystis jamaicensis, n. sp. from redtailed hawks (Buteo jamaicensis) definitive host and IFN- $\gamma$ gene knockout mice as experimental intermediate host. J Parasitol 103(5):555-564. https://doi.org/10.1645/17-10 